\title{
Improving data integrity in regulated bioanalysis: proposal for a generic data transfer process for LC-MS from the European Bioanalysis Forum
}

\author{
Cecilia Arfvidsson ${ }^{1}$, David Van Bedaf², Susanne Globig ${ }^{3}$, Magnus Knutsson ${ }^{4}$, Mark Lewis ${ }^{5}$, \\ Stuart McDougall ${ }^{6}$, Marco Michi ${ }^{7} \&$ Philip Timmerman*,8 \\ ${ }^{1}$ Clinical Pharmacology \& Quantitative Pharmacology, Clinical Pharmacology \& Safety Sciences, R\&D, AstraZeneca, Gothenburg, \\ Sweden \\ ${ }^{2}$ Nonclinical Safety, Janssen R\&D, Beerse, Belgium \\ ${ }^{3}$ Drug Metabolism \& Pharmacokinetics, Idorsia Pharmaceuticals Ltd, Allschwil, Switzerland \\ ${ }^{4}$ Bioanalysis, Ferring, Copenhagen, Denmark \\ ${ }^{5}$ Bioanalysis Immunogenicity \& Biomarkers, GlaxoSmithKline R\&D, Ware, UK \\ ${ }^{6}$ Bioanalytical Services, ARCinova, Alnwick, UK \\ ${ }^{7}$ Pharmacokinetics \& Drug Metabolism, Menarini Ricerche S.p.A., Pomezia, Italy \\ ${ }^{8}$ European Bioanalysis Forum vzw (EBF), Havenlaan 86c b204, 1000 Brussel, Belgium \\ *Author for correspondence: chair@e-b-f.eu
}

In this paper, the European Bioanalysis Forum reports back from the discussions with software developers, involved in regulated bioanalysis software solutions, on agreeing to data transfer specification in the bioanalytical labs' LC-MS workflows as part of today's Data Integrity (DI) challenges. The proposed specifications aim at identifying what consists of a minimum dataset, that is, which are the pre-identified fields to be included in DI proof bidirectional data transfer between LC-MS and information management systems. The proposal is an attempt from the European Bioanalysis Forum to facilitate new software solutions becoming available to increase compliance related to DI in today's LC-MS workflows. The proposal may also serve as a template and inspiration for new data transfer solutions in other workflows.

First draft submitted: 18 May 2020; Accepted for publication: 4 June 2020; Published online: 22 July 2020

Data Integrity (DI) is an important element to consider in ensuring the reliability and accuracy of (meta-) data generated in a regulated bioanalysis laboratory. In accordance with the 'ALCOA' principles [1], the data and information must be attributable, legible, contemporaneous, original and accurate, in addition to being complete. The critical importance of DI has been discussed both within the European Bioanalysis Forum (EBF) e-environment team and at several EBF Open Symposia over the last few years [2]. The interest and focus were fueled, among others, by several EBF member companies having received regulatory findings relating to DI issues during health authority inspections.

In a workshop on DI, arranged at the 11th EBF Open Symposium in Barcelona [3], the Medicines and Health products Regulatory Agency provided a detailed insight into their updated Guidance on GxP DI [4] and presented their view on how to best implement the aforementioned DI guidance. A summary from this workshop has been reported in previous EBF publication [5].

The format of the workshop at the 11th Open Symposium invited all delegates to actively participate in small interactive 'round table' discussions and openly share some of their current day-to-day experiences, challenges and frustrations related to data transfers. These round table discussions mainly focused on the bidirectional data transfers between LC-MS and information management (IM) systems (e.g., LIMS and ERP), with the lack of vendor neutral interface functionalities to ensure compliant data transfers being raised as a major concern. The discussions also highlighted a gap between the health authorities DI expectations and the availability of software that meets these expectations. It was suggested that the bioanalytical community should push for enhanced DI functionalities by making DI one of the main areas of focus when evaluating a new platform and/or instrument, 
thereby stimulating software developers, that is, the instrument vendor organizations, to prioritize DI controls when developing new software solutions. Finally, the delegates stressed the value of enhanced interaction and dialog between the bioanalytical community and software developers to specifically facilitate an improvement in the data transfer solutions currently offered.

\section{Building common understanding for future system solutions}

To address the identified gaps between the bioanalytical laboratory's DI needs in the LC-MS workflows, the EBF invited multiple software developers to take part in the preparation activities for a second e-environment focused workshop, arranged at the 12th EBF Open Symposium in November 2019 [6]. The purpose of this workshop was to connect software developers, Pharma/CRO bioanalytical laboratories and regulatory authorities together. Despite good discussions on the DI challenges at multiple meetings, the lack of any tangible progress to introduce new interface solutions continued to cause frustration. Current products still force the bioanalytical laboratories into manual and time-consuming quality-control steps to mitigate the DI risks. With the increased number of citings during health authority inspections over the last few years, the message is clear: DI issues must be resolved!

Having all parties gathered in the same room in a workshop setting, the EBF hoped to stimulate more solutionoriented discussions, the outcome of which was a clear message, that is:

- From the bioanalytical community: the software developers need to explain what they require from the bioanalytical community in order to 'make it happen';

- From the software developers: the bioanalytical community needs to agree on a joint request for the software developers to focus their efforts.

Today's situation: data life cycle

Building on the discussions and feedback from the workshop arranged at the 11th Open Symposium, a couple of short descriptions of 'Today's Situation' were presented to set the scene for the discussions at the 12th Open Symposium workshop and to highlight the current key challenges when data must be transferred in a compliant way.

Different DI challenges in today's LC-MS workflows were presented in the different steps of the data life cycle process, such as during:

- Sample receipt and preparation: linking the samples to the sample manifest and creating a run list from a sample manifest;

- Sample analysis: recording all relevant instrument settings from the sample/96-well plate to the detector;

- Data transfer and processing: making sure that the acquired data cannot be changed or deleted without a record in an audit trail;

- Reporting: secure data transfer to enable reporting;

- Archiving: ensuring all relevant data can be safely archived? And remains accessible, ensuring long-term readability.

Today's situation: data transfers from the IM system to the LC-MS system

All major software developers state with great confidence that their software is $100 \%$ compliant. True, but, in this statement they may only consider the performance when using their software 'in isolation' and, for obvious reasons, cannot consider all applications in which their software is used. As such, the 'interface landscape' has become the key issue for most bioanalytical labs and workflows when it comes to DI. The purpose of the short 'Today's Situation' descriptions, presented as a starting point for the workshop discussions, was to visualize the missing functionality in data transfer between the IM and the LC-MS system, without any judgement on any specific software solution, and where/how the software developers may contribute to help improve the current situation. Our focus was a joint mission to resolve the current interface and secure data transfer issues, considering previous challenges as positive input into the discussions.

As illustrated in Figure 1, the network area folder often used today could be protected against file modifications, but this requires an ad hoc configuration for each project/study, set up by the Information Technology department. After acquisition and processing of data a local export file (in text file [.txt] format) is often created. However, this can be saved at any location and modified without any audit trail before the file is saved on the network area. Manual quality control checks of the data transferred (ranging from spot check all the way to $100 \%$ check of the raw data) are therefore performed to ensure the integrity of the data. 


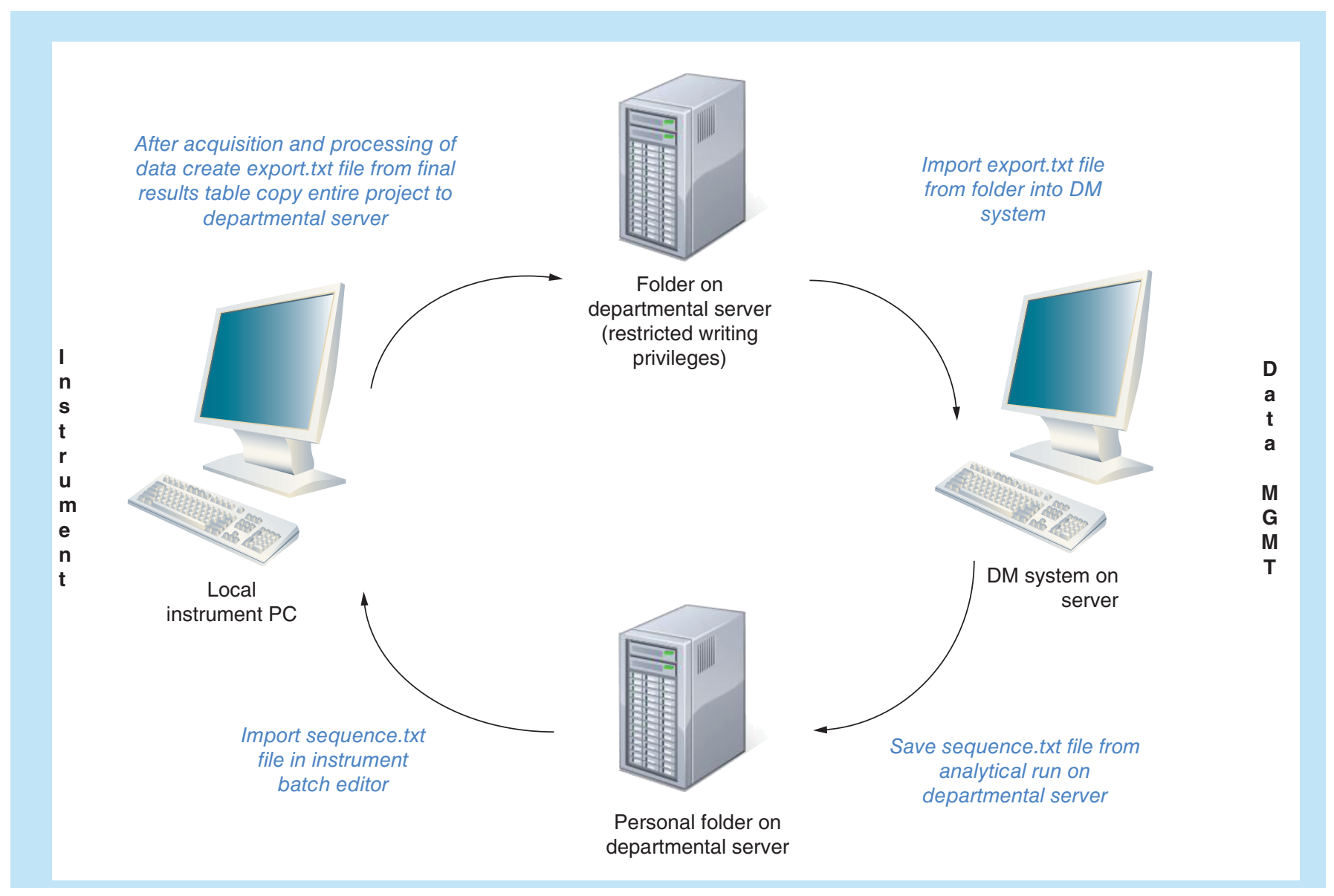

Figure 1. A common data transfer cycle in today's bioanalytical workflows: highlighting a Data Integrity gap due to the lack of a vendor independent secure data transfer solution.

The message from the instrument vendors back to the bioanalytical community at the workshop was clear and concise: in order to introduce a vendor independent solution, the bioanalytical community must agree on one generic data transfer specification. The agreed specification should provide the necessary clarity on the data fields to be included in secure data transfers and be applicable for any format in which the data is then transferred.

\section{Generic data transfer specification: an EBF proposal to facilitate in a secure data transfer solution}

Both software developers and the bioanalytical community are in full agreement of what the long-term solution for secure data transfers should look like: a file-less transfer of the complete dataset, using an interface that is compatible with all instrumentation and software, independent of brand.

Despite several attempts and ongoing discussions within various initiatives, such as the Allotrope foundation [7], a comprehensive long-term solution to support the bioanalytical needs is likely to be several years away. Customized solutions can indeed be available, but they will take time and be expensive to set-up. Such a venture will require robust project management to keep on track. EBF is, in this context, a relatively small community, acting only in the noncompetitive space for the benefit of the entire bioanalytical community, which may be an advantage in the discussion of a limited but sustainable solution that could be readily available and of benefit for all. Based on our discussions with various software developers, an improved secure data transfer solution may not be very far away, but only if the dataset can be limited to a minimum and jointly agreed number of fields. In a first important step toward a generic and compliant data transfer solution, the EBF is in this proposal focusing on the vendor neutral bidirectional data transfer between LC-MS and IM systems. In light of the current regulatory inspection feedback 
on DI, the bioanalytical community are keen to actively help seek a solution to address any DI gaps and support a stepwise approach to reach the final long-term secure data transfer solution, starting with the LC-MS workflow.

Since every bioanalytical laboratory (unfortunately) has its own workflows or inherent proprietary flavor, the complexity in the data format to be transferred easily accelerates into a complex dataset with an array of (likely noncritical) metadata. The generic data transfer specification proposed by the EBF below therefore only focuses on the dataset strictly required and includes those fields that are essential to safeguard DI. As emphasized by the software developers, it is critical to have an agreement in the bioanalytical community, on which fields to include to avoid the need for multiple customized solutions. Deciding which fields to include was made by assessing the risks and impact, and, if the integrity of the data was compromised. All fields included in the proposed specification should, however, be nonmandatory to allow for each laboratory to only select the fields/data that are relevant for their workflows and procedures.

With LC-MS being the primary platform for small molecules bioanalysis and with most companies expanding the use into peptides and proteins, our focus on LC-MS-IM interface has many immediate benefits: primarily reducing the time and effort required to manually perform quality control of data transfer. The proposal should also be seen as a move away from the current status quo and to showcase that progress can be made relatively simple if and when all agree on a common standard. In continuation, these initial mitigations could also energize other ongoing discussions and initiatives.

The generic vendor independent fields proposed to be included in the run/batch/worklist transfer from the IM system to the LC-MS are:

- Run/File specific fields

- Study/project

- Run/batch file

- User good laboratory practices (GLP)

- Plate barcode

- File name

- Sample specific fields

- Sample name/ID

- Sample barcode/ID

- Analyte(s)/internal standard(s) name

- Dilution factor

- Order number

- Plate position

- Sample type

- Concentration

Similar, the generic vendor-independent fields proposed to be included in the data transfer from the LC-MS to IM system are:

- Run/File specific

- Study

- Run

- Date/time

- User

- Plate barcode

- File name

- User comments

- Sample specific

- Sample name/ID 
- Sample barcode/ID

- Analyte(s)/internal standard(s) name

- Analyte raw data (peak area, peak height, ratio, etc.)

- Analyte(s) concentrations data

$\circ \quad$ Dilution factor

- Plate position

In the absence of any generic file-less interface solution, data must be secured using a secure file transfer solution, moving away from the current txt-file transfer approach. With an agreed specification, the EBF hopes to stimulate continued communication between the software developers to identify a secure technical file transfer solution.

\section{Concluding remarks \& future plans}

The two well-attended workshops on the e-environment topic, organized by the EBF e-environment team at the 11th and 12th EBF Open Symposia, highlighted the great interest from the bioanalytical community on the topic as well as its critical importance. Nevertheless, the progress for having new, easy access and generic/vendor-neutral solutions for secure data transfer tools continues to be slow. The workshop at the 12th Open Symposium was a successful first step toward a consensus and opened the opportunity for an increased dialog between the software developers and the bioanalytical community. Not only because of the dynamic discussions, but also as the workshop created the opportunity for interaction/dialog with multiple software developers to share their observations that a robust and less time/effort consuming secure data transfer process does is not far away if the bioanalytical community can agree on a standard set of (required) fields to be included in a generic data transfer specification.

This paper is the result of the continued discussion internally in the EBF e-environment team and subsequent discussion with software developers. We are ready to propose a generic data transfer specification format for the bidirectional data transfers between the LC-MS and IM systems for the software developers to work on. If achieved and being mindful for now that it focuses on LC-MS-IM bidirectional transfers only, we consider the collaboration practices and the implementation to be an important step to solve the DI challenges in the bioanalytical lab. It may not be the long-term solution for secure data transfers we ultimately need, but it can lead the way and showcase that quick wins can be made through increased interactions and dialog between industry and software developers. On the other hand, this proposal is also intended to continue our communication with the health authorities, highlighting that a long-term solution is still not readily available but that the bioanalytical community stays committed to continue to engage with software developers to proactively address the DI gaps and avoid further forced measures and process modifications due to inspection findings.

We hope that the current proposal can be an inspiration to facilitate additional steps, in which additional platforms can be considered. Multiple questions and challenges will have to be addressed and resolved as the complexity increase, that is, how to handle the various raw data and concentration outputs from the different plate-reader instrumentation? How to define rules for exceptions? And so on.

A continuation on the software developer engagement and dialog between all relevant parties continues to be critical to reach further progress toward the long-term DI solution, and as ever, as per the EBF mission [2], we are ready to help, stimulate or coordinate the similar solution-driven discussions as part of our day-to-day activities or at our workshops and symposia.

\section{Acknowledgments}

The EBF e-environment team would like to send a special thanks to G De Boer (Thermo Fisher Scientific), D Abramowitz (Sciex), N Loftus (Shimadzu), A Timmerman (Waters) and B Schaefer (BSSN Software) for their great engagement and input into these discussions. All workshop participants and EBF core members taking part in the discussions are also acknowledged.

\section{Disclaimer}

The views and conclusions presented in this paper are those of the European Bioanalysis Forum and do not necessarily reflect the representative affiliation or company's position on the subject. 


\section{Financial \& competing interests disclosure}

The authors have no relevant affiliations or financial involvement with any organization or entity with a financial interest in or financial conflict with the subject matter or materials discussed in the manuscript. This includes employment, consultancies, honoraria, stock ownership or options, expert testimony, grants or patents received or pending, or royalties.

No writing assistance was utilized in the production of this manuscript.

\section{References}

1. WHO. Guidance on Good Data and Record Management Practices (2016).

https://www.who.int/medicines/publications/pharmprep/WHO_TRS_996_annex05.pdf?ua=1

2. European Bioanalysis Forum. http://www.e-b-f.eu

3. 11th EBF Open Symposium slide decks. http://www.e-b-f.eu/bcn2018-slides/

4. Medicines \& Healthcare Products Regulatory Agency. ‘GXP' Data Integrity Guidance and Definitions (2018).

https://assets.publishing.service.gov.uk/government/uploads/system/uploads/attachment_data/file/687246/MHRA_GxP_data_integrity _guide_March_edited_Final.pdf

5. Arfvidsson C, Van Bedaf D, Doig M et al. Data integrity in regulated bioanalysis: a summary from the European Bioanalysis Forum Workshop in collaboration with the MHRA. Bioanalysis 11(13), 1227-1231 (2019).

6. 12th EBF Open Symposium slide decks. http://www.e-b-f.eu/ben2019-slides/

7. Allotrope Foundation. https://www.allotrope.org/ 